\title{
Multicomponent DNA vaccine-encoding Toxoplasma gondii GRA1 and SAG1 primes: anti-Toxoplasma immune response in mice
}

\author{
Xiao-Nan Wu • Jie Lin • Xu Lin • Jie Chen • \\ Zhong-Long Chen $\cdot$ Jian-Yin Lin
}

Received: 18 June 2012 / Accepted: 10 July 2012 /Published online: 27 July 2012

(C) The Author(s) 2012. This article is published with open access at Springerlink.com

\begin{abstract}
A multicomponent DNA vaccine, encoding Toxoplasma gondii GRA1 and SAG1, was constructed and tested for its ability to confer protection. BALB/c mice were challenged with tachyzoites of the virulent $T$. gondii $\mathrm{RH}$ strain at 4 weeks following the last immunization, and immune responses and survival times were observed. The results show that vaccination by the multicomponent vaccine prolonged survival of mice challenged with the $T$. gondii RH strain (from average $4.50 \pm 0.22$ to $7.60 \pm$ 0.74 days); induced high levels of IgG antibody (from $0.252 \pm 0.080$ to $0.790 \pm 0.083$ ), IFN-gamma (from $598.74 \pm$ 67.50 to $853.77 \pm 66.74 \mathrm{pg} / \mathrm{ml}$ ), and IL-2 (from $89.44 \pm 10.66$ to $192.24 \pm 19.90 \mathrm{pg} / \mathrm{ml}$ ); changed the $\mathrm{CD}^{+} / \mathrm{CD}^{+}$lymphocyte ratio (from $1.81 \pm 0.14$ to $1.09 \pm 0.19$ ); and stimulated NK cell-killing activity (from $46.81 \pm 3.96$ to $64.15 \pm$ $7.71 \%$ ). These findings demonstrate that a multicomponent DNA vaccine, encoding GRA1 and SAG1, primes a strong humoral and cellular immune response and enhances protection against $T$. gondii challenge. The new, combined DNA vaccine provides another means to combat $T$. gondii infection.
\end{abstract}

Jie Lin and Xiao-Nan Wu contributed equally to this work.

X.-N. Wu $\cdot$ J. Lin $\cdot X$. Lin $\cdot$ J.-Y. Lin $(\bowtie)$

Key Laboratory of Ministry of Education for Gastrointestinal

Cancer, Research Center of Molecular Medicine,

Fujian Medical University,

Fuzhou 350004, People's Republic of China

e-mail: jylin@mail.fjmu.edu.cn

X.-N. Wu $\cdot$ J. Chen $\cdot$ Z.-L. Chen

Public Health School, Fujian Medical University,

Fuzhou 350004, People's Republic of China

J. Lin

Fujian Center for Disease Control and Prevention,

Fuzhou 350001, People's Republic of China

\section{Introduction}

Toxoplasma gondii is an intracellular protozoan parasite that causes significant morbidity and mortality in congenitally infected and immunocompromised individuals. In humans, this relatively benign infection may reactivate under conditions of immunosuppression, such as in HIV-infected individuals and cancer patients, resulting in Toxoplasma encephalitis and other complications (Calabrese et al. 2008; Kato et al. 2005; Scorza et al. 2003). T. gondii infection acquired by pregnant women and its transmission to the fetus continue to be the cause of tragic yet preventable disease (Montoya and Remington 2008), and it is associated with transplacental infection. In veterinary medicine, $T$. gondii infection has economic impact by inducing abortion and neonatal loss in sheep and goats and is a source of transmission to humans (Dubey 1990). Therefore, the development of an effective vaccine against $T$. gondii would be of great value to both human and veterinary medicine.

Recently, there have been efforts to develop anti-T. gondii vaccines (Bhopale 2003). Among the new approaches, DNA vaccines have become a focus since they elicit potent, long-lasting humoral and cell-mediated immunity (Alarcon et al. 1999). Membrane-associated surface antigens (SAG1 and SAG2), secreted dense-granule proteins (GRA1, GRA2, GRA4, and GRA7), rhoptry proteins (ROP1 and ROP2), and micronemal proteins (MIC1, MIC2, MIC3, MIC4, and MIC8) are all putative $T$. gondii vaccine candidates (Dautu et al. 2007; Fang et al. 2009; Jongert et al. 2007; Li et al. 2011; Liu et al. 2010, 2009; Wang et al. 2009; Xue et al. 2008; Zhang et al. 2007). These antigens have shown certain protection, increased survival time of animals, and a reduced number of brain cysts in rodents. Also, employment of different forms of adjuvant was evaluated and compared the effects on the immune response stimulated by DNA vaccine (Khosroshahi et al. 2012). 
SAG1 is the best-characterized candidate vaccine. Previous studies have shown that DNA vaccines with SAG1 induce both humoral and cellular immune responses and partial protection against $T$. gondii (Couper et al. 2003; Fachado et al. 2003; Liu et al. 2009; Mevelec et al. 2005; Xue et al. 2008; Zhang et al. 2007). Dense-granule antigens (GRA), secreted in abundance, are major components of both the vacuole-surrounding tachyzoites and the cyst wall-surrounding slower growing bradyzoites (CesbronDelauw 1994). Therefore, the GRAs may be important protective antigens. Among the GRAs, GRA1, a product of $T$. gondii tachyzoites and bradyzoites, is a promising candidate. A GRA1 DNA vaccine can prime an antiToxoplasma immune response (Bivas-Benita et al. 2003; Jongert et al. 2007; Vercammen et al. 2000). In addition, native protein encoded by GRA1 is a type of $\mathrm{Ca}^{2+}$-binding protein that functions to activate or stabilize the reticulum structure and may also function as a $\mathrm{Ca}^{2+}$ buffer (Lin et al. 2010). GRA1 and SAG1 possess distinct antigenicity, and their expression spans different stages of $T$. gondii development. Therefore, the objectives of this study were to construct eukaryotic expression plasmids, pVAX1-GRA1 and pVAX1-SAG1, and to evaluate the immune response and protective effect of a combined DNA vaccine in BALB/c mice against challenge with a highly lethal $T$. gondii $\mathrm{RH}$ strain. The results show that a multicomponent DNA vaccine, encoding $T$. gondii GRA1 and SAG1, primed a strong humoral and cellular immune response and enhanced protection against $T$. gondii challenge.

\section{Materials and methods}

Cell lines, plasmids, mice, and reagents

Raw264.7 cell, a murine macrophage cell line, was obtained from the Shanghai Cell Biological Institute (Shanghai, China). Eukaryotic expression vector pVAX1 was purchased from Invitrogen (Carlsbad, CA, USA). SPF grade BALB/c mice were purchased from Shanghai SLAC Laboratory Animal Co., Ltd. (Shanghai, China).

Taq DNA polymerase (high fidelity) was purchased from Stratagene (Santa Clara, CA, USA). Restriction enzymes (EcoR I and Kpn I), calf intestine alkaline phosphatase, and T4 DNA Ligase were purchased from New England Biolabs (Beverly MA, USA). Endonuclease-Free Plasmid Mega kit was from QIAGEN (Hilden, Germany). Liposome (Lipofectamine 2000), and kanamycin were obtained from Invitrogen. Mouse anti-T. gondii SAG1 and rabbit anti-T. gondii trophozoite antibodies were from Biodesign (Saco ME, USA). 3-(4,5-Dimethylthiazol-2-yl)-2,5-diphenyltetrazolium (MTT) was obtained from Sigma (St. Louis, MO, USA). Fluorescein isothiocyanate (FITC)-labeled anti-mouse CD4 antibody, polyethylene (PE)-labeled anti-CD8 antibody was from Beckman Coulter (Shanghai, China). Detection kits for mouse IFN- $\gamma$, IL-2, and IL- 4 cytokines (OptEIA ${ }^{\mathrm{TM}}$ ) were from BD Bioscience Corp (Shanghai, China).

\section{Primers}

The following primers were used: GRA1VAXF, 5'CGG GGTACCATGGTGCGTGTGAGCGCTATTG (Kpn I); GRA 1 VAXR， 5' CCGGAATTCTTACTCTCTC TCTCCTGTTAGG (EcoR I); SAG1VAXF, 5' CGGGGTACCATGTCGGTTTCGCTGCACCAC (Kpn I); and SAG1VAXR, 5' CCGGAATTCTCACGCGACACAA GCTGCG (EcoR I).

\section{Amplification of GRA1 and SAG1 genes}

GRA1 or SAG1 was amplified from recombinant plasmid pCMV/myc-GRA1 or pCMV/myc-SAG1 (constructed in our previous project) templates, respectively. Briefly, samples were denatured for $5 \mathrm{~min}$ at $94{ }^{\circ} \mathrm{C}$, followed by 30 cycles of denaturation $\left(94^{\circ} \mathrm{C}, 30 \mathrm{~s}\right)$, with annealing $\left(55^{\circ} \mathrm{C}\right.$, $30 \mathrm{~s}$ ) and extension $\left(72{ }^{\circ} \mathrm{C}, 1 \mathrm{~min}\right)$, followed by a final extension at $72{ }^{\circ} \mathrm{C}$ for $10 \mathrm{~min}$. PCRs were performed with the primer pairs GRA1VAX F-R or SAG1VAX F-R.

Construction of the recombinant plasmids pVAX1-GRA1 and pVAX1-SAG1 and large-scale DNA preparation

PCR products were cloned into the $\mathrm{pVAX} 1$ vector. Sequencing was performed on an ABI PRISM genetic analyzer in Takara Biotechnology (Dalian, China) using primers T7 (F) and BGH (R). Sequence alignments were done using BLAST with default settings (PubMed, http://blast.ncbi.nlm.nih.gov). All plasmids were propagated in Escherichia coli DH5- $\alpha$.

The plasmids pVAX1-GRA1 and pVAX1-SAG1 were purified by Endo-Free Plasmid Mega kit prior to using for vaccination and dissolved in sterile endotoxin-free phosphate-buffered saline (PBS). Plasmid integrity was checked by agarose gel electrophoresis after digestion with appropriate restriction enzymes. The DNA concentration and purity was determined by $\mathrm{A}_{260}$.

Macrophage transfection by liposome and gene expression

One day before transfection, RAW264.7 was inoculated into six well plates (Corning Incorporated, Corning, NY, USA) with 2-ml common culture medium and cultured in $5 \% \mathrm{CO}_{2}$ at $37{ }^{\circ} \mathrm{C}$ to $80 \%$ confluence. Five micrograms DNA (pVAX1-GRA1, pVAX1-SAG1, or pVAX1) were diluted in $50-\mu \mathrm{l}$ sera-free medium $(10 \mu \mathrm{l}$ liposome diluted in 40 $\mu \mathrm{l}$ serum-free medium) and mixed. DNA and liposomes were incubated for $20 \mathrm{~min}$ at room temperature to form 
DNA-liposome complexes. Transfection mixture $(100 \mu \mathrm{l})$ was added to the macrophage culture and gently mixed. The plates were then incubated in $5 \% \mathrm{CO}_{2}$ at $37{ }^{\circ} \mathrm{C}$ for $48 \mathrm{~h}$.

GRA1 and SAG1 expression was detected by immunohistochemistry. Briefly, RAW264.7 (transfected by pVAX1GRA1, pVAX1-SAG1, empty control plasmid pVAX1, or nontransfected), grown on the cover slip, was fixed by prechilled $70 \%$ alcohol for $30 \mathrm{~min}$. Cells were rinsed three times with PBS for $3 \mathrm{~min}$, incubated with $50 \mu$ l peroxidase-blocking solution for $10 \mathrm{~min}$, and rinsed three times with PBS. Nonimmune serum was incubated for $10 \mathrm{~min}$, and the surplus was discarded. Primary antibody $(50 \mu \mathrm{l})$ was then incubated for $60 \mathrm{~min}$, rinsed three times with PBS, and followed by $50 \mu$ biotin-conjugated secondary antibody for $10 \mathrm{~min}$ and a rinse with PBS (three times). Complexes were visualized by incubation with $50 \mu \mathrm{l}$ streptavidin peroxidase solution for $10 \mathrm{~min}, \mathrm{PBS}$ rinse (three times), and addition of $100 \mu \mathrm{l}$ freshly prepared DAB for 3-10 min. Following a wash by double distilled water, samples were counterstained by hematoxylin and washed by PBS. Unless noted, all incubations and washes were performed at room temperature. Samples were photographed using the Leica photo system (Solms, Germany).

\section{Vaccination}

$\mathrm{BALB} / \mathrm{c}$ mice were randomly divided into five groups of 18 each. Plasmids were diluted to $2 \mu \mathrm{g} / \mu \mathrm{l}$ in PBS. The five groups were: $50 \mu \mathrm{l}$ PBS, $100 \mu \mathrm{g}$ empty pVAX1 vector, $100 \mu \mathrm{g}$ pVAX-GRA1, $100 \mu \mathrm{g}$ pVAX1-SAG1, and $50 \mu \mathrm{g}$ pVAX1-GRA1 + $50 \mu \mathrm{g}$ pVAX1-SAG1, respectively. Each group received three injections (separated by 2 -week intervals) in both tibias and anterior muscles. Injections were administered with a $0.3-\mathrm{ml}$ syringe.

\section{Challenge experiments}

Four weeks after the last vaccination, ten mice were selected randomly from each group and challenged by intraperitoneal injection with $10^{5}$ tachyzoites of $T$. gondii RH strain. The length of survival was recorded.

\section{Evaluation of the humoral immune response}

IgG antibodies were monitored by ELISA. The eyes were extirpated to get sera from mice 4 weeks after the third vaccination. Ninety-six-well plates were coated with tachyzoite lysis antigen of $T$. gondii. Serum samples were diluted $(1: 100)$ prior to testing.

Evaluation of the cellular immune response

ELISA was used to measure IFN- $\gamma$, IL-2, and IL-4 concentrations. The ratio of $\mathrm{CD} 4^{+} / \mathrm{CD}^{+}$in spleen cells was measured by Beckman Coulter XL flow cytometry (488 nm excitation, $620 \mathrm{~nm}$ emission for PE, and $525 \mathrm{~nm}$ for FITC), using $0.5 \times 10^{6}$ cells.

\section{NK cell-killing activity}

Splenocytes (effector cells) obtained from mice 4 weeks after the third vaccination were adjusted to $5 \times 10^{6} / \mathrm{ml}$ in RPMI 1640 with $5 \%$ fetal bovine serum. The eugenic Yac-1 cells (target cells) were adjusted to $2 \times 10^{5} / \mathrm{ml}$ in RPMI 1640. Effector and target cells were plated in $100 \mu \mathrm{l}$ 96-well flat-bottomed plates at E/T ratios of 25:1. Effector and target cells, alone in RPMI 1640, served as controls. All assays were done in triplicate. After a 44-h incubation at $5 \% \mathrm{CO}_{2}$ and $37{ }^{\circ} \mathrm{C}, 20 \mu \mathrm{MTT}$ was added to each well and further incubated at $5 \% \mathrm{CO}_{2}, 37^{\circ} \mathrm{C}$ for $4 \mathrm{~h}$. Excess supernatant was discarded, and $100 \mu 100 \%$ DMSO was added to each well, and mixed for $10 \mathrm{~min}$, and the $\mathrm{A}_{570}$ was measured. Cytotoxicity (in percent $)=[($ experimental - effector spontaneous) / target spontaneous $] \times 100$.

\section{Results}

The construction of DNA vaccines and identification and expression in macrophages

A 573-bp PCR product, corresponding to the GRA1 coding sequence, was generated, digested with the appropriate restriction enzymes, and cloned into the expression vector pVAX1, generating pVAX1-GRA1. A 960-bp PCR product for SAG1 was created the same way as for GRA1, generating pVAX1-SAG1. Sequence alignment for both GRA1 and SAG1 by PubMed verified that the sequence was correct. Figure 1 shows the identification of recombinant plasmid pVAX1-GRA1, pVAX1-SAG1 by PCR, and restriction enzyme digestion.

To determine whether the $T$. gondii GRA1 and SAG1 proteins were expressed in RAW264.7, following transfection with pVAX1-GRA1, pVAX1-SAG1, or an empty vector, expression was tested by immunohistochemistry. Brown-stained particles were found in the cytoplasm that had been transfected by pVAX1-GRA1 or pVAX1-SAG1. No stain was observed in cells transfected with control vector pVAX1 (Fig. 2).

Protective effect of GRA1 and SAG1 DNA vaccine in BALB/c mice challenged with $T$. gondii

To test whether the DNA vaccine, encoding $T$. gondii GRA1 and SAG1, could protect against a lethal T. gondii infection, BALB/c mice were immunized with $100 \mu \mathrm{g}$ pVAX1 empty vector, $100 \mu \mathrm{g}$ pVAX-GRA1, $100 \mu \mathrm{g}$ pVAX1-SAG1, or 


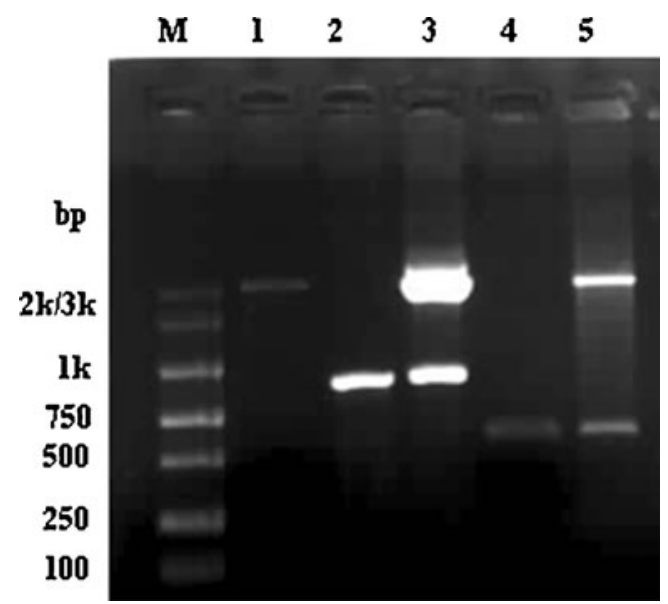

Fig. 1 Characterization of recombinant plasmids pVAX1-GRA1 and pVAX1-SAG1 by PCR and restriction enzyme digestion. $M$ DNA marker, 1 pVAX1, 2 amplified fragment of SAG1 gene, 3 recombinant plasmid pVAX1-SAG1 digested by Kpn I and EcoR I, 4 amplified fragment of GRA1 gene, 5 recombinant plasmid pVAX1-GRA1 digested by Kpn I and EcoR I

$50 \mu \mathrm{g}$ pVAX1-GRA1 + $50 \mu \mathrm{g}$ pVAX1-SAG1, respectively. The survival time is shown in Fig. 3. Control mice died beginning on day 3 , and all mice had died by day 5 . The mice lived on average, just $4.50 \pm 0.22$ days, following infection. Mice immunized with pVAX1 died beginning on day 3 , and all had died by day 6 , with an average of $4.80 \pm$ 0.29 days. The mice of the pVAX1-GRA1 group died beginning on day 3 , and all had died by day 5 , with an average of $4.10 \pm 0.28$ days. The mice of the pVAX1-SAG1 group also began to die from the third day on, and all had died by day 8 , (average $=4.70 \pm 0.60$ days). Mice immunized with pVAX1-GRA1-SAG1 began to die beginning on day 4; however, three mice were still alive on day 10 (average $=$ $7.60 \pm 0.74$ days). This last group lived longer than the other groups $(P<0.05)$. The statistical significance between different groups was demonstrated by survival analysis (logrank statistic, Table 1). The data indicate that multicomponent DNA vaccines, encoding $T$. gondii GRA1 and SAG1, can increase the survival time of mice challenged with $T$. gondii RH strain.

\section{Anti-Toxoplasma IgG levels in BALB/c mice}

To evaluate the immunogenicity of the GRA1 and SAG1 DNA vaccines, BALB/c mice were immunized with empty vector or single- or two-gene vaccines. Mice were bled, and anti-Toxoplasma IgG levels were determined by ELISA 4 weeks following the last vaccination (Fig. 4). The antiToxoplasma IgG optical density (OD) value was $0.790 \pm$ 0.083 in the pVAX1-GRA1 + pVAX1-SAG1 group, while the values were $0.430 \pm 0.052$ and $0.451 \pm 0.060$ in pVAX1GRA1 and pVAX1-SAG1 groups, respectively. The OD values were $0.310 \pm 0.071$ in the control vector group and $0.252 \pm 0.080$ for the PBS control. Using ANOVA statistical analysis, the OD values in the pVAX1-GRA1 + pVAX1SAG1, pVAX1-GRA1, and pVAX1-SAG1 were significantly higher than those in PBS control group $(P<0.05)$. The values for the pVAX1-GRA1 + pVAX1-SAG1 were higher
Fig. 2 Expression of GRA1/

SAG1 antigen in mouse

macrophage

(immunocytochemistry, $\times 400$ ).

Upper left RAW264.7, upper

right $\mathrm{RAW} 264.7$ transfected

with pVAX1, lower left

RAW264.7 transfected with pVAX1-SAG1, lower right RAW264.7 transfected with pVAX1-GRA1
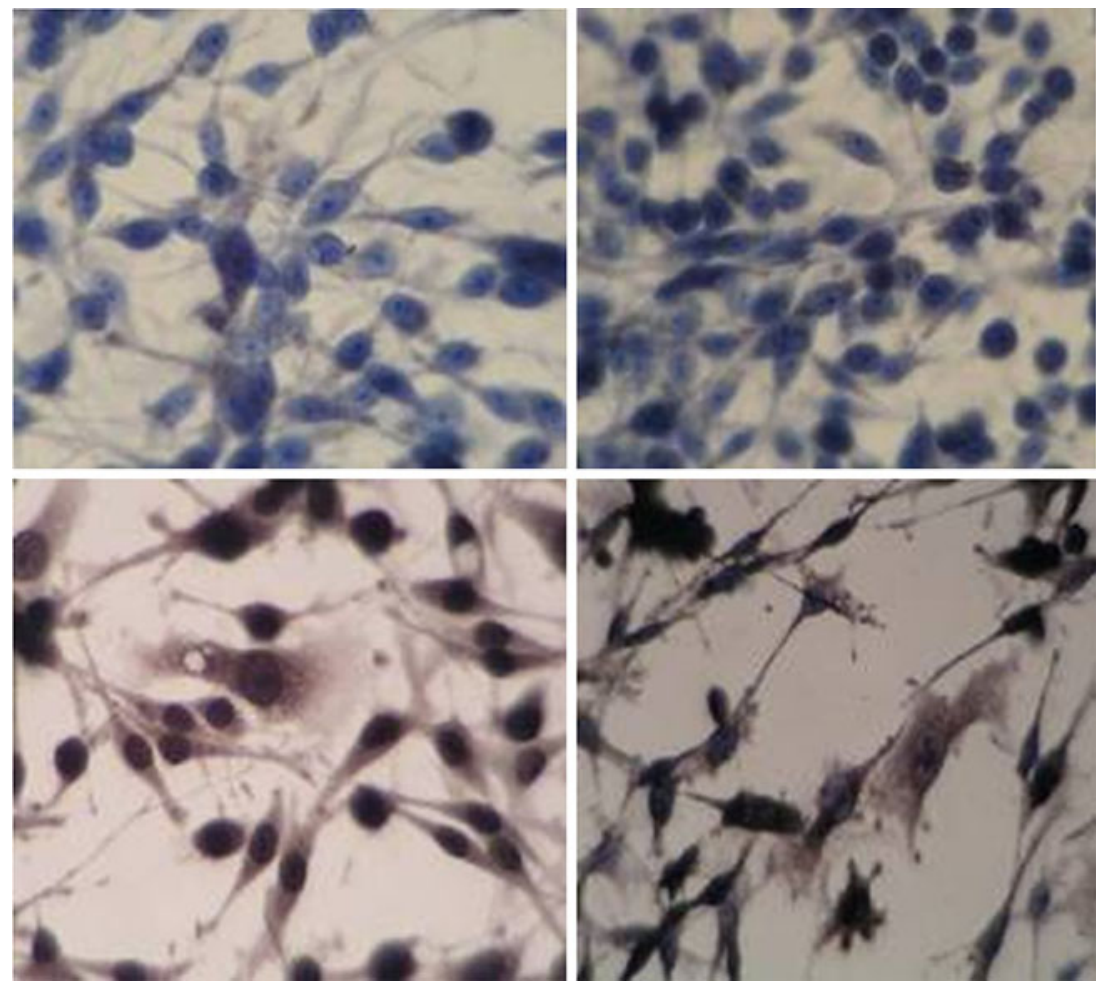
Fig. 3 Survival curves of BALB/c mice injected with $50 \mu$ PBS or immunized with $100 \mu \mathrm{g}$ empty pVAX1, $100 \mu \mathrm{g}$ pVAX-GRA1, $100 \mu \mathrm{g}$ pVAX1SAG1, $50 \mu \mathrm{g}$ pVAX1-GRA1 + $50 \mu \mathrm{g}$ pVAX1-SAG1, respectively, and challenged with $10^{5}$ tachyzoites of $T$. gondii $\mathrm{RH}$ strain 4 weeks after last vaccination. Each group contained ten mice. Survival data were recorded until the end of the observation period

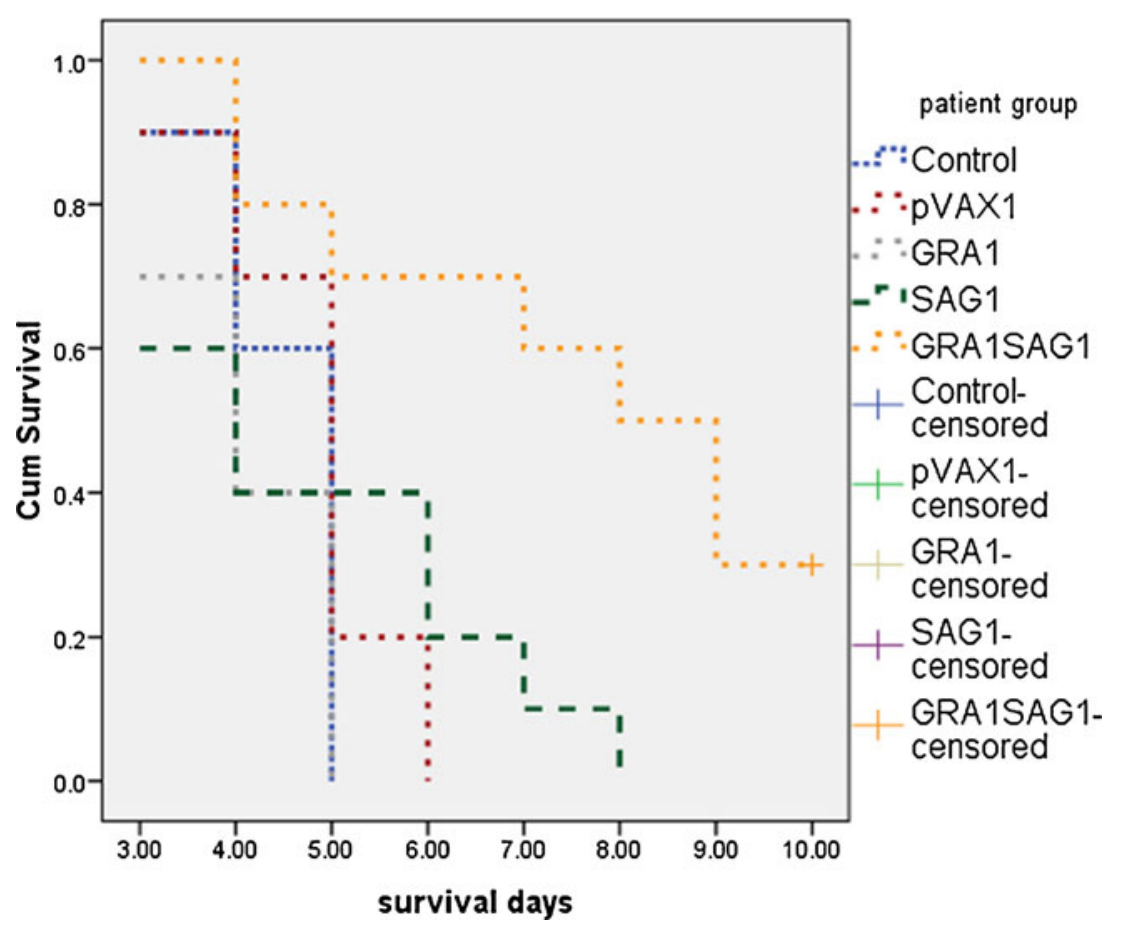

than those for pVAX1-SAG1 and pVAX1-GRA1 $(P<0.05)$. These results indicate that a multicomponent DNA vaccine, encoding $T$. gondii GRA1 and SAG1, induces a strong antiT. gondii $\mathrm{IgG}$ antibody response.

Production of IFN- $\gamma$, IL-2, and IL-4 in sera from BALB/c mice

To evaluate the role of cytokines in the cellular immune response against $T$. gondii infection, IFN- $\gamma$, IL-2, and IL-4 serum levels in immunized mice were measured by ELISA (Fig. 5). The IFN- $\gamma$ levels in the pVAX1-GRA1 + pVAX1SAG1 (853.77 \pm 66.74 pg/ml), pVAX1 (679.63 \pm 44.79 pg/ $\mathrm{ml})$, and pVAX1-SAG1 (669.78 $\pm 50.42 \mathrm{pg} / \mathrm{ml})$ groups were higher relative to those of control group $(598.74 \pm 67.50 \mathrm{pg} /$ ml) $(P<0.05)$. The IFN- $\gamma$ levels in the pVAX1-GRA1 + pVAX1-SAG1 group were elevated relative to those of pVAX1-GRA1 and pVAX1-SAG1 $(P<0.05)$. IL-2 levels in the pVAX1-GRA1+ pVAX1-SAG1 group (192.24
$19.90 \mathrm{pg} / \mathrm{ml})$ were higher than those of empty pVAX1 vector $(92.77 \pm 13.94 \mathrm{pg} / \mathrm{ml})$ and PBS control $(89.44 \pm$ $10.66 \mathrm{pg} / \mathrm{ml})(P<0.05)$. In contrast, the IL-4 levels of the five groups were similar $(P>0.05)$. The data show that the DNA vaccine, encoding GRA1 and SAG1, is able to produce higher levels of IFN- $\gamma$ and IL-2.

Percent change of $\mathrm{CD}^{+} / \mathrm{CD}^{+} \mathrm{T}$ cells in splenocytes from $\mathrm{BALB} / \mathrm{c}$ mice immunized with DNA vaccines

Protection against $T$. gondii is dependent on both $\mathrm{CD} 4^{+}$and $\mathrm{CD}^{+}$cells ( $\mathrm{Li}$ et al. 2010). To evaluate the role of $\mathrm{CD} 4^{+}$and $\mathrm{CD}^{+}$cells in the cellular immune response against $T$. gondii challenge, the percent change of $\mathrm{CD}^{+} / \mathrm{CD}^{+} \mathrm{T}$ cells in splenocytes from immunized mice was measured by flow cytometry (Table 2). Compared with PBS control, the percent of $\mathrm{CD}^{+}$cells in the pVAX1-GRA1 + pVAX1-SAG1 $(37.42 \pm 4.84 \%)$, pVAX1-SAG1 (44.32 $\pm 2.61 \%)$, and pVAX1-GRA1 $(45.82 \pm 3.01 \%)$ groups was reduced $(P<$

Table 1 Results of log-rank statistics for survival time of BALB/c mice challenged with $10^{5}$ tachyzoites of $T$. gondii RH strain 4 weeks after last vaccination $(N=10)$

\begin{tabular}{lccccc}
\hline Group & Control & pVAX1 & pVAX1-GRA1 & pVAX1-SAG1 & pVAX1-GRA1 + pVAX1-SAG1 \\
\hline Control & - & $1.07(0.3000)$ & $1.04(0.3090)$ & $0.33(0.5680)$ & $7.97(0.0048)$ \\
pVAX1 & $1.07(0.3000)$ & - & $2.98(0.0840)$ & $0.12(0.7263)$ & $8.11(0.0044)$ \\
pVAX1-GRA1 & $1.04(0.3090)$ & $2.98(0.0840)$ & - & $1.26(0.2620)$ & $9.97(0.0016)$ \\
pVAX1-SAG1 & $0.33(0.5680)$ & $0.12(0.7263)$ & $1.26(0.2620)$ & - & $8.10(0.0044)$ \\
pVAX1-GRA1 + pVAX1-SAG1 & $7.97(0.0048)$ & $8.11(0.0044)$ & $9.97(0.0016)$ & $8.10(0.0044)$ & - \\
\hline
\end{tabular}

The values in the table are chi-square values tested by log-rank statistic of pairwise comparisons. The values in parentheses are significance values 
Fig. 4 Levels of $\operatorname{IgG}$ antibody in sera from $\mathrm{BALB} / \mathrm{c}$ mice immunized with single- or twogene DNA vaccines, empty vector, or PBS (control group) as measured by ELISA 4 weeks after last vaccination. Each group was composed of eight mice

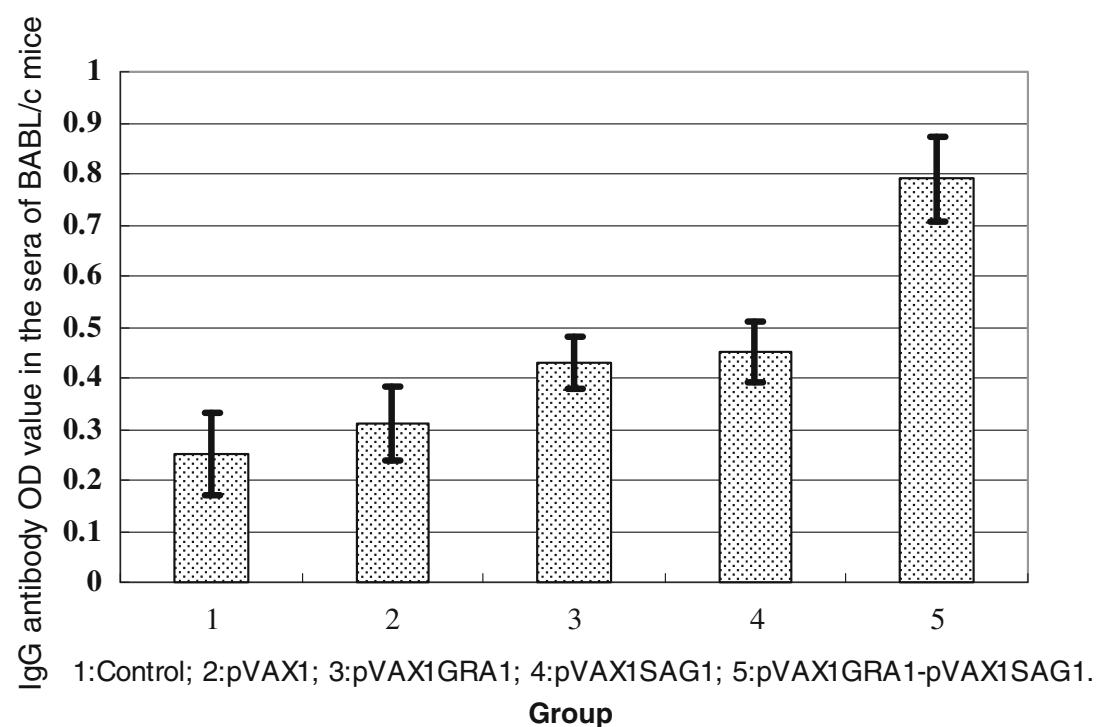

0.05). The percent of $\mathrm{CD}^{+}$cells in the pVAX1-GRA1 + pVAX1-SAG1 group $(34.94 \pm 5.30 \%)$ increased relative to that of pVAX1 or PBS control $(P<0.05)$. The $\mathrm{CD} 4^{+} / \mathrm{CD} 8^{+}$ ratio of pVAX1-GRA1 + pVAX1-SAG1 $(1.09 \pm 0.19 \%)$, pVAX1-SAG1 $(1.56 \pm 0.22 \%)$, and pVAX1-GRA1 $(1.66 \pm$ $0.13 \%)$ groups decreased relative to that of pVAX1 $(P<$ $0.05)$. The $\mathrm{CD}^{+} / \mathrm{CD}^{+}$ratio of pVAX1-GRA $1+$ pVAX1SAG1 was lower than that observed for the pVAX1-GRA1 and pVAX1-SAG1 groups $(P<0.05)$.

NK cell-killing activity in BALB/c splenocytes from mice immunized with DNA vaccine

To test whether NK cells play an important role in the cellular immune responses induced by the GRA1 and SAG1 vaccine, the NK cell-killing activity of splenocytes was determined by MTT assays (Fig. 6). The NK cell-killing rate of splenocytes of pVAX1-GRA1 + pVAX1-SAG1, the pVAX1-GRA1, the
pVAX1-SAG1, the pVAX1 empty vector, and the PBS control group was $64.15 \pm 7.71,52.03 \pm 6.96,55.95 \pm 7.37,48.13 \pm$ 4.58 , and $46.81 \pm 3.96 \%$, respectively. ANOVA analysis indicated that the increased killing of the pVAX1-GRA1 + pVAX1-SAG1, pVAX1-GRA1, and pVAX1-SAG1 groups were statistically significant relevant to the control group $(P$ $<0.05)$. The killing rate of the pVAX1-GRA1 + pVAX1SAG1 NK cells was higher than that of the pVAX1-GRA1 and pVAX1-SAG1 NK cells $(P<0.05)$.

\section{Discussion}

The potential for a multicomponent DNA vaccine, encoding $T$. gondii GRA1 and SAG1 to induce protective immune responses in BALB/c mice, was investigated. The results indicate that the vaccine primes humoral and cellular responses and enhances protection against $T$. gondii challenge.
Fig. 5 Levels of IFN- $\gamma$, IL-2, and IL-4 in sera from the BALB/c mice immunized with the single- or two-gene DNA vaccine, the empty vector, or PBS (control) as measured by ELISA 4 weeks following the last vaccination. Each group was composed of eight mice

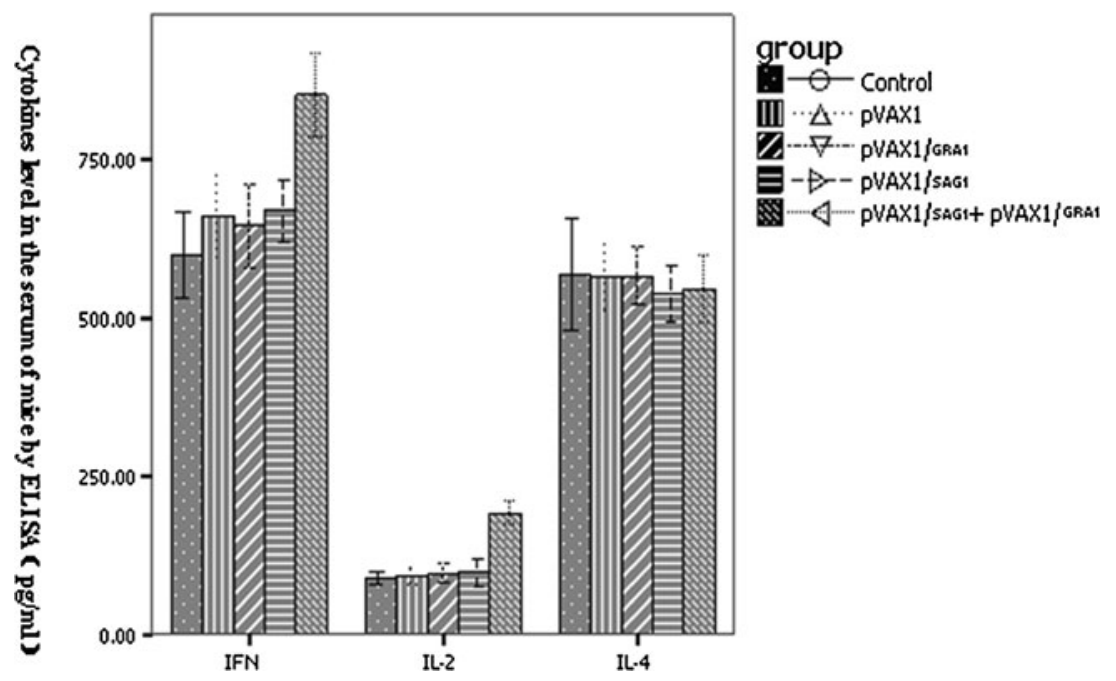


Table 2 Flow cytometry analysis of T lymphocyte subsets in splenocytes from BALB/c mice 4 weeks after last vaccination $(N=8)$

${ }^{\mathrm{a}}$ Compared with the PBS control group, $P<0.05$

${ }^{\mathrm{b}}$ Compared with the $\mathrm{pVAX} 1$ group, $P<0.05$

\begin{tabular}{llll} 
Group & \multicolumn{2}{l}{ T 1ymphocyte subsets } & \\
\cline { 2 - 4 } & $\mathrm{CD}^{+}(\%)$ & $\mathrm{CD}^{+}(\%)$ & $\mathrm{CD}^{+} / \mathrm{CD}^{+}$ \\
\hline PBS control & $48.59 \pm 1.85$ & $26.91 \pm 2.12$ & $1.81 \pm 0.14$ \\
pVAX1 & $48.01 \pm 2.32$ & $26.35 \pm 1.69$ & $1.83 \pm 0.12$ \\
pVAX1-GRA1 & $45.82 \pm 3.01^{\mathrm{a}}$ & $27.82 \pm 3.24$ & $1.66 \pm 0.13^{\mathrm{b}}$ \\
pVAX1-SAG1 & $44.32 \pm 2.61^{\mathrm{a}, \mathrm{b}}$ & $28.89 \pm 4.87$ & $1.56 \pm 0.22^{\mathrm{a}, \mathrm{b}}$ \\
pVAX1-GRA1 + pVAX1-SAG1 & $37.42 \pm 4.84^{\mathrm{a}, \mathrm{b}}$ & $34.94 \pm 5.30^{\mathrm{a}, \mathrm{b}}$ & $1.09 \pm 0.19^{\mathrm{a}, \mathrm{b}}$ \\
\hline
\end{tabular}

The challenge dose may be a factor that influences the observed protection level. In this study, we sought to assess vaccine protection against a high-dose challenge (with regard to mouse) because humans are not considered to be as sensitive to Toxoplasma as mouse, especially at the lethal dose. The lethal dose of $10^{5} \mathrm{RH}$ parasites in mouse (Fachado et al. 2003) may not be lethal if the equivalent were used in humans. Interestingly, even at this high-dose level, three mice vaccinated with the multicomponent vaccine were still alive on day 10; however, all control mice had died by day 5 , and statistical significance was observed. The results suggest that combined immunization of pVAX1-GRA1 and pVAX1-SAG1 can significantly prolong survival of mice challenged with $T$. gondii $\mathrm{RH}$ strain, compared with the control. There was not an obvious prolonged survival of mice challenged with $T$. gondii RH strain when immunized with plasmid pVAX1-GRA1 or pVAX1-SAG1 alone. These results suggest that a combined GRA1 and SAG1 can enhance a protective effect against a lethal T. gondii infection, consistent with current studies ( $\mathrm{Li}$ et al. 2010; Liu et al. 2009; Wang et al. 2009).

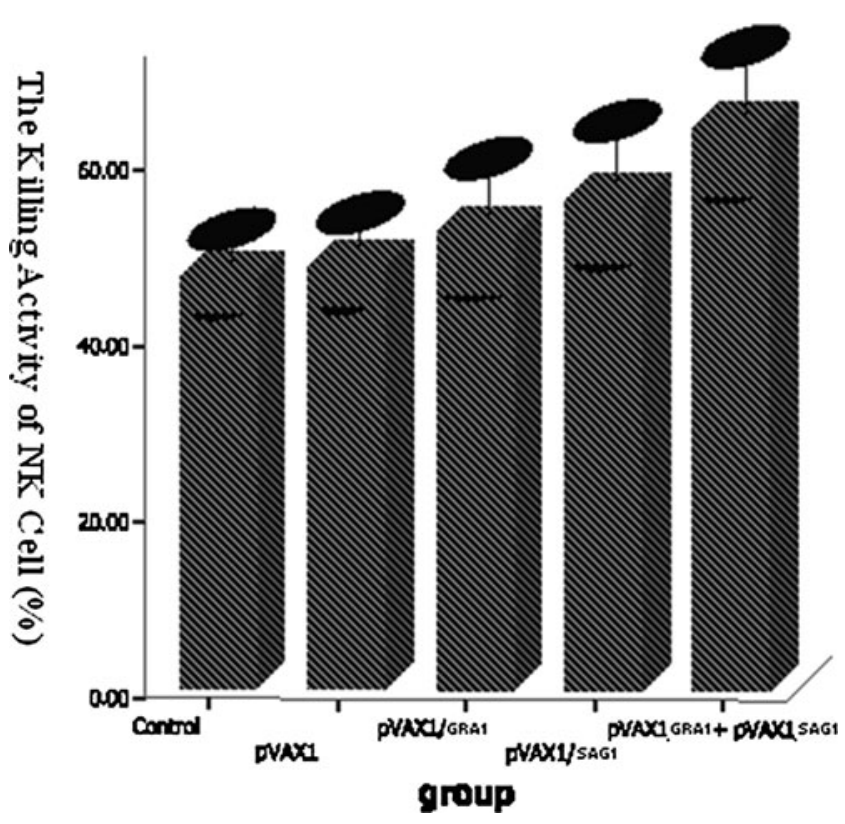

Fig. 6 The NK cell-killing activity of splenocytes from BALB/c mice 4 weeks after the last vaccination. Each group was composed of eight mice
To explore the mechanisms of protective immunity induced by the DNA vaccine encoding GRA1 and SAG1, humoral and cellular immune responses were analyzed. For humoral immune responses, our data showed that the relative level of anti-T. gondii IgG antibody in mice immunized with pVAX1-GRA1 or pVAX1-SAG1 was elevated, comparing to controls, and it was further elevated by immunization with pVAX1-GRA1 + pVAX1-SAG1, suggesting that a DNA vaccine, encoding GRA1 and SAG1, could produce stronger humoral immunity.

It has been shown that protection against $T$. gondii is dependent on both $\mathrm{CD}^{+}$and $\mathrm{CD}^{+}$cells ( $\mathrm{Li}$ et al. 2010). $\mathrm{CD}^{+} \mathrm{T}$ cells constitute the major $\mathrm{T}$ cell subset involved in acquired cellular immune protection against $T$. gondii (Tan et al. 2011). In vivo $T$ cell depletion experiments indicate that $\mathrm{CD}^{+} \mathrm{T}$ cells are essential for survival of GRA1-vaccinated $\mathrm{C} 3 \mathrm{H}$ mice during acute phase of $T$. gondii infection, while depletion of $\mathrm{CD}^{+} \mathrm{T}$ cells led to an increase in brain cyst burden during the chronic phase of infection (Scorza et al. 2003). We observed that 4 weeks after the last vaccination, the percentage of splenic $\mathrm{CD}^{+} \mathrm{T}$ cells in immunized mice increased in the pVAX1-GRA1 + pVAX1-SAG1 group, while pVAX1-GRA1- and pVAX1-SAG1-immunized mice did not exhibit any significant change. The $\mathrm{CD} 4^{+} / \mathrm{CD}^{+}$ratio was reduced significantly. These data suggest that the immune protection against $T$. gondii challenge is elicited by multicomponent pVAX1-GRA1 + pVAX1-SAG1, and that it is mediated primarily through induction of $\mathrm{CD}^{+} \mathrm{T}$ subsets and a Th1-type cellular immune response.

NK cells are a kind of lymphocyte with powerful cytotoxicity and play an important role in innate immunity. We found that NK cell-killing activity of pVAX1-GRA1 + pVAX1-SAG1, pVAX1-SAG1, and pVAX1-GRA1 groups were all higher than that of controls, while the cytotoxicity of the pVAX1-GRA1 + pVAX1-SAG1 group was higher than that of pVAX1-GRA1 and pVAX1-SAG1, suggesting that combined GRA1 and SAG1 is more effective at enhancing NK cell activity.

Cytokines play an important role in Th cell function. Previous studies have shown that IFN- $\gamma$ can promote Th1 differentiation, and that IL-4 can induce development of Th2 cells (Maggi et al. 1992). Th1 cells can further stimulate 
macrophages and CTL through IFN- $\gamma$, while Th2 cells regulate B cell helper activities by IL-4. Furthermore, IFN- $\gamma$ and IL-2 are important stimulatory cytokines involved in the protection against parasitic infection. T cells and NK cells are the primary producers of IFN- $\gamma$, which enhances NK cell-killing activity and activates mononuclear macrophages to kill $T$. gondii intracellularly. IL-2 can activate the cytotoxic activity of $\mathrm{T}$ cells and facilitate production of various cytokines, such as IFN- $\gamma$. IL-2 also stimulates NK cell cytotoxicity, while IL-4 is thought to downregulate the inflammatory immune response (Matowicka-Karna et al. 2009; Suzuki et al. 1989, 1988). To evaluate the effects of vaccine pVAX1-GRA1 and pVAX1-SAG1, three cytokines were monitored. Our results indicate that $p$ VAX1-GRA1 and pVAX1-SAG1 are capable of stimulating high levels of IFN- $\gamma$ and IL-2, although IL-4 levels were similar across all groups, suggesting that vaccination was preferentially driving a Th1-type response. The empty $\mathrm{pVAX} 1$ plasmid appeared to also stimulate IFN $-\gamma$ secretion, suggesting that the DNA macromolecule itself may function as an immunological adjuvant and induce nonspecific stimulation on the immune system.

In summary, our results demonstrate that a multicomponent DNA vaccine, encoding T. gondii GRA1 and SAG1, primes the immune system to generate a stronger humoral and cellular immune response and enhances protection against a $T$. gondii challenge. This combination vaccine may, therefore, provide a more effective means to combat troublesome $T$. gondii infections.

Acknowledgments This work was supported by the National Natural Science Foundation of China (number 30940062).

Open Access This article is distributed under the terms of the Creative Commons Attribution License which permits any use, distribution, and reproduction in any medium, provided the original author(s) and the source are credited.

\section{References}

Alarcon JB, Waine GW, McManus DP (1999) DNA vaccines: technology and application as anti-parasite and anti-microbial agents. Adv Parasitol 42:343-410

Bhopale GM (2003) Development of a vaccine for toxoplasmosis: current status. Microbes Infect 5:457-462

Bivas-Benita M, Laloup M et al (2003) Generation of Toxoplasma gondii GRA1 protein and DNA vaccine loaded chitosan particles: preparation, characterization, and preliminary in vivo studies. Int J Pharm 266:17-27

Calabrese KS, Tedesco RC et al (2008) Serum and aqueous humour cytokine response and histopathological alterations during ocular Toxoplasma gondii infection in C57BL/6 mice. Micron 39:13351341

Cesbron-Delauw MF (1994) Dense-granule organelles of Toxoplasma gondii: their role in the host-parasite relationship. Parasitol Today 10:293-296
Couper KN, Nielsen HV et al (2003) DNA vaccination with the immunodominant tachyzoite surface antigen (SAG-1) protects against adult acquired Toxoplasma gondii infection but does not prevent maternofoetal transmission. Vaccine 21:28132820

Dautu G, Munyaka B, Carmen G et al (2007) Toxoplasma gondii: DNA vaccination with genes encoding antigens MIC2, M2AP, AMA1 and BAG1 and evaluation of their immunogenic potential. Exp Parasitol 116:273-282

Dubey JP (1990) Status of toxoplasmosis in sheep and goats in the United States. J Am Vet Med Assoc 196:259-262

Fachado A, Rodriguez A et al (2003) Protective effect of a naked DNA vaccine cocktail against lethal toxoplasmosis in mice. Vaccine 21:1327-1335

Fang R, Nie H, Wang $Z$ et al (2009) Protective immune response in $\mathrm{BALB} / \mathrm{c}$ mice induced by a suicidal DNA vaccine of the MIC3 gene of Toxoplasma gondii. Vet Parasitol 164:134-140

Jongert E, de Craeye S, Dewit J et al (2007) GRA7 provides protective immunity in cocktail DNA vaccines against Toxoplasma gondii. Parasite Immunol 29:445-453

Kato M, Claveria FG et al (2005) Toxoplasma gondii antigens GRA1 (p24) and SAG1 (p30): a comparison of their stimulatory influence on T-cell activation and cytokine expression in in vitro cultures. Pathobiology 72:160-164

Khosroshahi KH, Ghaffarifar F, Sharifi Z et al (2012) Comparing the effect of IL-12 genetic adjuvant and alum non-genetic adjuvant on the efficiency of the cocktail DNA vaccine containing plasmids encoding SAG-1 and ROP-2 of Toxoplasma gondii. Parasitol Res. doi:10.1007/s00436-012-2852-7

Li B, Oledzka G, McFarlane RG et al (2010) Immunological response of sheep to injections of plasmids encoding Toxoplasma gondii SAG1 and ROP1 genes. Parasite Immunol 32:671-683

Li WS, Chen QX, Ye JX et al (2011) Comparative evaluation of immunization with recombinant protein and plasmid DNA vaccines of fusion antigen ROP2 and SAG1 from Toxoplasma gondii in mice: cellular and humoral immune responses. Parasitol Res 109:637-644

Liu S, Shi L, Cheng YB et al (2009) Evaluation of protective effect of multi-epitope DNA vaccine encoding six antigen segments of Toxoplasma gondii in mice. Parasitol Res 105:267-274

Liu MM, Yuan ZG, Peng GH et al (2010) Toxoplasma gondii microneme protein 8 (MIC8) is a potential vaccine candidate against toxoplasmosis. Parasitol Res 106:1079-1084

Lin J, Lin X et al (2010) Toxoplasma gondii: expression of GRA1 gene in endoplasmic reticulum promotes both growth and adherence and modulates intracellular calcium release in macrophages. Exp Parasitol 125:165-171

Maggi E, Parronchi P, Manetti R et al (1992) Reciprocal regulatory effects of IFN-gamma and IL-4 on the in vitro development of human Th1 and Th2 clones. J Immunol 148:2142-2147

Matowicka-Karna J, Dymicka-Piekarska V et al (2009) Does Toxoplasma gondii infection affect the levels of $\mathrm{IgE}$ and cytokines (IL5, IL-6, IL-10, IL-12, and TNF-alpha)? Clin Dev Immunol 2009:374-696

Mevelec MN, Bout D et al (2005) Evaluation of protective effect of DNA vaccination with genes encoding antigens GRA4 and SAG1 associated with GM-CSF plasmid, against acute, chronical and congenital toxoplasmosis in mice. Vaccine 23:4489-4499

Montoya JG, Remington JS (2008) Management of Toxoplasma gondii infection during pregnancy. Clin Infect Dis 47:554-566

Scorza T, D'Souza S et al (2003) A GRA1 DNA vaccine primes cytolytic CD8(+) T cells to control acute Toxoplasma gondii infection. Infect Immun 71:309-316

Suzuki Y, Orellana MA et al (1988) Interferon-gamma: the major mediator of resistance against Toxoplasma gondii. Science 240:516-518 
Suzuki Y, Conley FK et al (1989) Importance of endogenous IFNgamma for prevention of toxoplasmic encephalitis in mice. J Immunol 143:2045-2050

Tan F, Hu X, Luo FJ et al (2011) Induction of protective Th1 immune responses in mice by vaccination with recombinant Toxoplasma gondii nucleoside triphosphate hydrolase-II. Vaccine 29:2742-2748

Vercammen M, Scorza T et al (2000) DNA vaccination with genes encoding Toxoplasma gondii antigens GRA1, GRA7, and ROP2 induces partially protective immunity against lethal challenge in mice. Infect Immun 68:38-45
Wang H, He S, Yao Y et al (2009) Toxoplasma gondii: protective effect of an intranasal SAG1 and MIC4 DNA vaccine in mice. Exp Parasitol 122:226-232

Xue M, He S, Cui Y et al (2008) Evaluation of the immune response elicited by multi-antigenic DNA vaccine expressing SAG1, ROP2 and GRA2 against Toxoplasma gondii. Parasitol Int 57:424-429

Zhang J, He S, Jiang H et al (2007) Evaluation of the immune response induced by multiantigenic DNA vaccine encoding SAG1 and ROP2 of Toxoplasma gondii and the adjuvant properties of murine interleukin-12 plasmid in BALB/c mice. Parasitol Res 101:331-338 